\title{
Comparação entre Homens e Mulheres Hipercolesterolêmicos de Alto Risco de Desenvolvimento de Aterosclerose. Estudo dos Fatores de Risco e da Resposta ao Tratamento com Pravastatina
}

\author{
Raul D. Santos, Raul C. Maranhão e pesquisadores
}

São Paulo, SP

\begin{abstract}
Objetivo - Comparar, entre homens e mulheres hipercolesterolêmicos, a prevalência dos fatores de risco e a resposta ao tratamento com pravastatina.

Métodos - Avaliados 486 homens e 368 mulheres, sendo 230 homens e 187 mulheres, que receberam $10 \mathrm{mg}$ de pravastatina por 3 meses.

Resultados - Houve diferença respectivamente entre mulheres e homens em relação a: hipertensão arterial (45,5\% vs 40,8\%; $p=0,0012)$, hipertrofia ventricular esquerda (33,0\% vs 22,0\%; $p=0,0041)$, sedentarismo (94,8\% vs 87,8\%; $p=0,0005)$, tabagismo $(43,0 \%$ vs $61,8 \%$; $p<0,0001)$, escores de Framingham $(20,0 \pm 7,1$ vs $16,0 \pm 7,6 p<0,001), H D L-C(43,0 \pm 11,0 v s 38,0 \pm 9,0 m g / d L$; $p<0,001)$, triglicérides $-T G(216,0 \pm 115,0$ vs $271,0 \pm$ $172,0 \mathrm{mg} / \mathrm{dL} ; \mathrm{p}<0,001)$ e indices de Castelli (IC) I e II

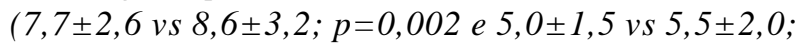
$p=0,015)$. Sob ação da pravastatina, houve maior redução nos TG (32,0 vs $21,0 \%$ p <0,05) e IC I $(-41,0 \%$ vs $-37,0 \%$; $p<0,05)$ e II $(-40,0$ vs $-38,0 \%$; $p<0,05)$ nos homens.
\end{abstract}

Conclusão - Homens e mulheres diferiram nos fatores de risco e resposta ao tratamento com a pravastatina.

Palavraschave: hipercolesterolemia, aterosclerose, fatores de risco

\section{Comparison of Hypercholesterolemic Men and Women at High Risk for Atherosclerosis. Study of Risk Factors and Response to Pravastatin Treatment}

Purpose - To compare the prevalence of risk factors, and the response to pravastatin treatment between men and women.

Methods - We evaluated 486 men and 386 women, of these 230 men and 187 women received 10mg of pravastatin for three months.

Results - There were differences between women and men in respectively: arterial hypertension $(45.5 \% \mathrm{vs}$ $40.8 \% ; p=0.0012)$, left ventricular hypertrophy (33.0\% vs $22.0 \% ; p=0.0041)$, sedentarism $(94.8 \%$ vs $87.8 \%$; $p=0.0005)$, smoking $(43.0 \%$ vs $61.8 \%$; $p<0.0001)$, Framingham scores (20.0 7.1 vs $16.0 \pm 7.6 p<0.001), H D L$ $C(43.0 \pm 11.0$ vs $38.0 \pm 9.0 \mathrm{mg} / \mathrm{dL} ; p<0.001)$, triglycerides$T G(216.0 \pm 115.0$ vs $271.0 \pm 172.0 \mathrm{mg} / \mathrm{dL} ; \mathrm{p}<0.001)$ and

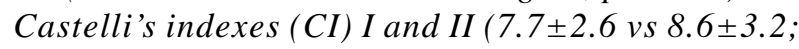
$p=0.002$ and $5.0 \pm 1.5$ vs 5.5 $\pm 2.0 ; p=0.015$ ). In men under pravastatin treatment there was a greater reduction in $T G$ (32.0 vs $21.0 \% p<0.05)$ and CI I $(-41.0 \%$ vs $-37.0 \%$; $p<0.05)$ and II (-40.0vs $-38.0 \%$; $p<0.05)$.

Conclusion - Men and women differed in risk factors prevalence and response to treatment with pravastatin.

Key-words: hypercholesterolemia, atherosclerosis, risk factors

Arq Bras Cardiol, volume 70 (nº 6), 383-387, 1998

Instituto do Coração do Hospital das Clínicas - FMUSP

Correspondência: Raul D. Santos - Incor - Av. Dr. Eneas C. Aguiar, 44 - $1^{\circ}$ - $05403-$ 000 - São Paulo, SP

Recebido para publicação em 21/1/98

Aceito em 26/3/98
No Brasil, as doenças cardiovasculares são a principal causa de morbidade e mortalidade em ambos os sexos. Comparando-se os coeficientes de mortalidade por doenças cardiovasculares de algumas cidades brasileiras com a de outros países, encontramos taxas proporcionalmente mais elevadas no sexo feminino ${ }^{1,2}$. Além disso, ocorre taxa de 
mortalidade por doença cérebro-vascular relativamente mais elevada nas mulheres brasileiras ${ }^{3}$. A origem desses achados poderia ser explicada pela alta prevalência dos fatores de risco na população feminina brasileira, associada a um mau controle desses fatores ${ }^{2}$. Dentre os principais, podemos citar a hipertensão arterial sistêmica (HAS), o tabagismo, a obesidade e, por fim, a hipercolesterolemia.

O objetivo deste estudo foi comparar as diferenças entre os fatores de risco para aterosclerose entre homens e mulheres hipercolesterolêmicos. Avaliamos também se houve diferença entre os sexos, a curto prazo, na resposta ao tratamento hipolipemiante com o inibidor da HMGCo-A redutase pravastatina. Os pacientes avaliados foram considerados de alto risco para doença cardiovascular, tomandose por base o critério de apresentarem pelo menos três fatores de risco para doença aterosclerótica.

\section{Métodos}

Participaram do estudo 486 homens e 368 mulheres, originários de consultórios de 334 cardiologistas, seguidos durante 1996. Os critérios de inclusão no estudo foram: 1) idade entre 18 e 75 anos; 2) mulheres sem potencial de gravidez ou em uso de métodos adequados de anticoncepção. Além disso, deveriam apresentar resultado negativo de teste para gravidez no soro ou na urina dois dias antes do tratamento; 3) hipercolesterolemia definida como colesterol total do plasma (CT), acima de $260 \mathrm{mg} / \mathrm{dL}$ na seleção, e $\geq 240 \mathrm{mg} / \mathrm{dL}$ após quatro semanas de dieta fase I da Associação Americana do Coração; 4) indivíduos com presença de pelo menos dois outros fatores de risco para doença cardiovascular, além da hipercolesterolemia, como: a) HAS; b) diabetes mellitus tipo I ou II; c) sexo masculino > 45 anos; d) tabagismo; e) inatividade física; f) obesidade (índice de massa corpórea $>30 \mathrm{~kg} / \mathrm{m} 2$ ); g) hipertrofia ventricular esquerda. Os critérios de exclusão foram: 1) indivíduos analfabetos ou de baixo nível sócio econômico nos quais o tratamento proposto pudesse ser prejudicado; 2) mulheres grávidas, em amamentação ou com potencial de gravidez (não estando em uso de métodos adequados de contracepção); 3) dependência do álcool (duas ou mais doses por dia); 4) infarto do miocárdio (IM) ou acidente vascular cerebral (AVC) nos últimos três meses; 5) presença de hepatopatias, colelitíase sintomática, nefropatias, pancreatite crônica, doenças da tireóide, diabetes mellitus descontrolado (glicemia de jejum >140mg/dL) ou outras doenças que, segundo o médico investigador ou a bula da pravastatina, pudessem colocar os indivíduos sob risco ou interferir no resultado do estudo; 6) HAS não controlada; 7) uso concomitante de hormônios, sais biliares, imunossupressores ou drogas hipolipemiantes; 8) alergia ou eventos adversos sérios decorrentes do uso prévio de inibidores da HMGCo-A redutase. Foi obtido consentimento informado para participação neste estudo em $100 \%$ dos indivíduos.

Protocolo do estudo - Foram realizadas cinco consultas médicas em intervalo de quatro semanas (total 20 semanas).

Consulta 0 (pré estudo) - Os participantes foram pré- selecionados com base em seu perfil lipídico prévio e orientados, verbalmente, em dieta pobre em gorduras saturadas e em colesterol (dieta fase I da Associação Americana do Coração).

Consulta 1 - Constando de anamnese e exame físico completo com ênfase na medida de peso e pressão arterial (PA) com o indivíduo deitado e sentado. Foram analisados eletrocardiogramas (ECG) disponíveis para detecção de sobrecarga ventricular esquerda, assim como o perfil lipídico: colesterol total, colesterol da LDL (LDL-C), colesterol daHDL(HDL-C), colesterol da VLDL(VLDL-C)e triglicérides (TG) do plasma. Quando existiu dúvida em relação a distúrbios hepáticos ou neuromusculares foram determinadas as aminotransferases e a creatinoquinase (CPK). Foi calculado o escore de risco de desenvolvimento de doença coronária, baseado na tabela derivada do estudo de Framingham ${ }^{4}$, onde são atribuídos pontos para a idade, HDL-C, colesterol total, PA sistólica e presença de tabagismo, hipertrofia ventricular esquerda ou diabetes mellitus. $\mathrm{O}$ escore é baseado na soma destes fatores: quanto maior ele for, maior o risco de doença arterial coronária (DAC). Foram calculados também os índices de risco de Castelli I e II (CT/ HDL-C e LDL-C/HDL-C respectivamente) ${ }^{5}$. Todos os participantes receberam orientação verbal sobre dieta, fumo, exercício e hipertensão, além de folheto explicativo sobre dieta para reduzir o colesterol. Na consulta seguinte os pacientes receberam outros folhetos informativos sobre colesterol, estresse, exercício físico, fumo e um folheto geral sobre a saúde do coração.

Consulta 2 - Repetida a avaliação clínica e colhida amostra sangüínea para determinação do perfil lipídico e estabelecido que, caso os pacientes se mantivessem dentro dos critérios de inclusão, e caso concordassem em continuar no estudo, seria administrada a dose de $10 \mathrm{mg}$ de pravastatina em tomada única à noite $\left(\right.$ pravacol $^{\circledR}$, Laboratórios Bristol Myers Squibb do Brasil, São Paulo) durante quatro semanas. Do total de pacientes avaliados 230 homens e 187 mulheres receberam o medicamento. Houve perda do seguimento de nove homens e sete mulheres. Qualquer mudança ocorrida no tratamento medicamentoso dos participantes durante o protocolo foi anotada.

Consulta 3 - Repetido o exame clínico, mantendo-se $10 \mathrm{mg} /$ dia de pravastatina por mais quatro semanas, na ausência de efeitos colaterais clínicos.

Consulta 4 - Repetido o exame clínico e laboratorial das consultas 1 e 2 .

A determinação do perfil lipídico no plasma foi realizada em laboratórios de confiança dos investigadores, após jejum de $12 \mathrm{~h}$. O colesterol total, o HDL-C e os TG foram determinados por métodos enzimáticos. O LDL-colesterol foi calculado utilizando-se a fórmula de Friedewald para triglicérides (TG) de até $400 \mathrm{mg} / \mathrm{dL}^{6}$. Os médicos foram orientados para encaminhar os indivíduos aos mesmos laboratórios durante todo o estudo (cinco consultas). Se houvesse dúvida quanto aos resultados, os mesmos eram repetidos a critério de seu médico assistente. Caso necessário foram determinados também os níveis de aminotransferases e creatinoquinase $(\mathrm{CPK})$ por métodos cinéticos. 
Foi feita a comparação dos fatores de risco citados previamente, além do perfil lipídico, índices de Castelli e escores de Framingham e comparada a resposta de ambos os sexos no tocante ao peso, PA sistólica e diastólica, perfil lipídico, índices de Castelli I e II e escores de Framingham. Foi avaliada também a incidência de efeitos colaterais ao tratamento.

Foram considerados eventos adversos a ocorrência de doenças ou de sintomas e sinais que aparecessem ou piorassem no decorrer do estudo. Esta consideração foi feita independentemente dos investigadores acreditarem ou não no relacionamento com a droga do estudo. Foram consideradas três categorias de intensidade dos eventos: leve (sintomas ou sinais toleráveis), moderada (desconforto suficiente para interferir nas atividades habituais) e grave (incapacitante com inabilidade para desempenhar as atividades habituais). A avaliação dos eventos adversos foi feita pelo registro de sinais e sintomas clínicos, pelo seguimento das enzimas musculares quando necessário e pela necessidade de suspensão da pravastatina durante o estudo.

Com relação à análise estatística, nas tabelas, os dados são apresentados como média \pm desvio padrão da média. As variações dos parâmetros analisados mostram as variações das médias em percentual $(\Delta \%)$. As variáveis qualitativas foram analisadas pelo teste do qui-quadrado e as variáveis contínuas pelos testes t de Student e análise de medidas repetitivas. A significância foi aceita ao nível de 5\% (p<0,05).

\section{Resultados}

A média de idade das mulheres foi maior do que a dos

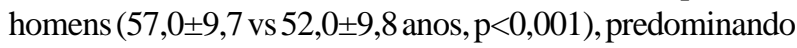
a HAS ( $\mathrm{p}=0,0012$ ), a hipertrofia ventricular esquerda $(\mathrm{p}=0,0041)$ e o sedentarismo $(\mathrm{p}=0,0005)$ (tab. I). A DAC foi diagnosticada em $11 \%$ dos homens e $7 \%$ das mulheres $(\mathrm{p}=0,06)$. Nos homens predominou o tabagismo $(\mathrm{p}<0,0001)$ (tab.I), assim comoníveis mais baixos de HDL-C $(\mathrm{p}<0,001) \mathrm{e}$ mais elevados de TG ( $<<0,001)$ (tab. II). Os escores de Framingham foram maiores nas mulheres do que nos homens $[(20,0 \pm 7,1$ vs $16,0 \pm 7,6)(p<0,001)]$. Os índices de Castelli I e II foram maiores nos homens do que nas mulheres [(respectivamente $8,6 \pm 3,2$ vs $7,7 \pm 2,6(\mathrm{p}=0,002)$ e $5,5 \pm 2,0$ vs $5,0 \pm 1,5 ; \mathrm{p}=0,015)]$. Com exceção de número pouco menor de portadores de sobrecarga ventricular esquerda ao ECG, (estatisticamente não significativo), os pacientes que rece-

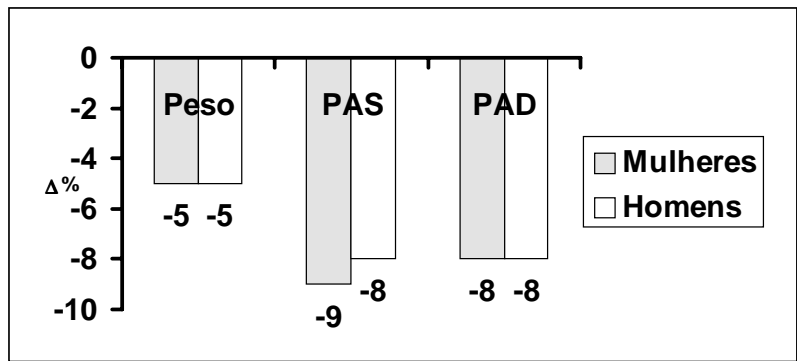

Fig. 1 - Variação percentual do peso, pressão arterial sistólica (PAS) e pressão arterial diastólica (PAD) em homens e mulheres durante seguimento de 16 semanas.

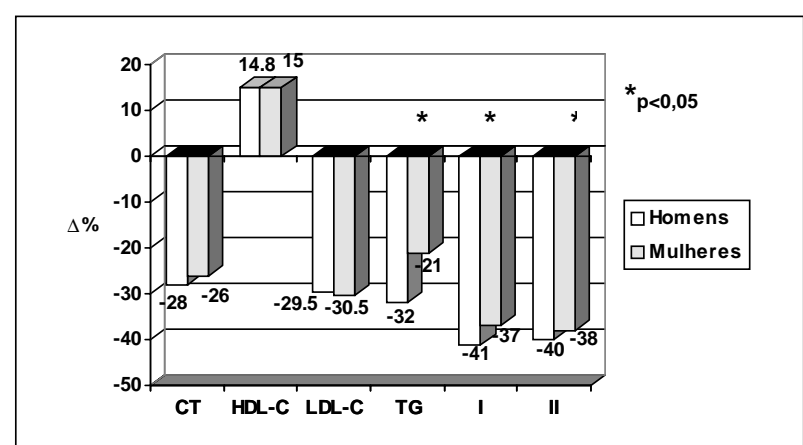

Fig. 2 - Variação percentual dos lípides e índices I e II de Castelli entre homens e mulheres após o tratamento com dieta hipolipemiante (4 semanas) e pravastatina (12 semanas). CT- colesterol total; HDL-C- colesterol da HDL; LDL-C- colesterol da LDL; TG- triglicérides do plasma; I- índice I de Castelli; II- índice II de Castelli.

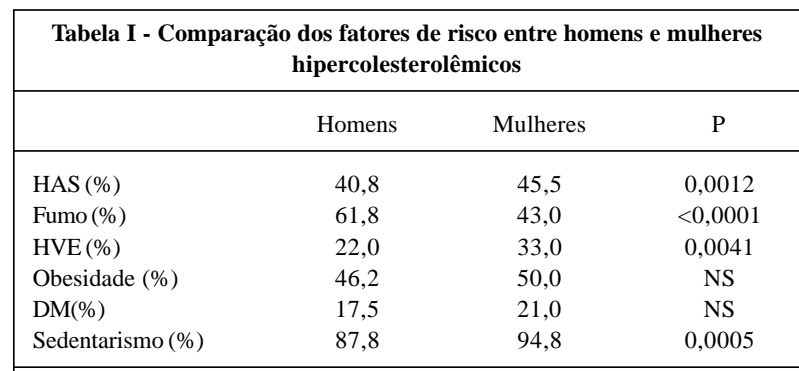

HAS - hipertensão arterial sistêmica; HVE - hipertrofia ventricular esquerda; DM - diabetes mellitus; NS - não significativo.

Tabela II - Perfil lipídocp e índices de Castelli de homens e mulheres hipercolesterolêmicos antes da terapia com pravastatina

\begin{tabular}{|lccc|}
\hline & Homens & Mulheres & P \\
\hline CT & $308,0 \pm 61,0$ & $306,0 \pm 48,0$ & NS \\
LDL-C & $198,0 \pm 47,0$ & $204,0 \pm 37,0$ & NS \\
HDL-C & $38,0 \pm 9,0$ & $43,0 \pm 11,0$ & $<0,001$ \\
TG & $271,0 \pm 172,0$ & $216,0 \pm 115,0$ & $<0,001$ \\
Índice I & $8,6 \pm 3,2$ & $7,7 \pm 2,6$ & 0,002 \\
Índice II & $5,5 \pm 2,0$ & $5,0 \pm 5$ & 0,015 \\
\hline
\end{tabular}

CT - colesterol total; HDL-C - colesterol da HDL; LDL-C - colesterol da LDL; TG-triglicérides do plasma; I - índice I de Castelli; II - índice II de Castelli.

\begin{tabular}{|lccc|}
\hline \multicolumn{2}{|c|}{$\begin{array}{c}\text { Tabela III - Eventos adversos em homens e mulheres diagnosticados } \\
\text { durante o seguimento de 16 semanas }\end{array}$} \\
\hline Queixa & Homens (\%) & Mulheres (\%) & p \\
\hline cefaléia & 7,8 & 7,5 & NS \\
Tontura & 7,0 & 6,4 & NS \\
Tosse & 7,0 & 8,5 & NS \\
Dor muscular & 4,3 & 4,3 & NS \\
Hipotensão postural & 1,7 & 1,0 & NS \\
Erupção cutânea / Prurido & 0,9 & 1,1 & NS \\
Náuseas/ vômitos & 3,9 & 7,0 & NS \\
Pirose & 1,7 & 3,7 & NS \\
Disfunção/ Sexual & 2,2 & 1,1 & NS \\
Outros & 0,43 & 0,5 & NS \\
\hline NS- não significativo. & & & \\
\hline
\end{tabular}

beram a pravastatina apresentaram proporcionalmente os mesmos fatores de risco do que o grupo total de pacientes (dados não mostrados).

Após quatro meses de tratamento, a resposta do peso e da PA sistêmica foi semelhante nos dois grupos. As redu- 
ções foram significativas em relação ao basal $(\mathrm{p}<0,0001)$ porém não diferiram entre os grupos (fig. 1). Os homens apresentaram maior redução média nos valores dos TG [(32,0 vs 21,0\% p <0,05)] assim como nos índices de Castelli I $[(-41,0 \%$ vs $-37,0 \%$ p $<0,05)]$ e II $[(-40,0$ vs $-38,0 \%)(\mathrm{p}<0,05)]$ (fig. 2). Não houve mudança significativa no tratamento medicamentoso no decorrer do estudo.

Não houve diferença significativa nas queixas de eventos adversos nos dois grupos de pacientes (tab. III). Estes foram considerados leves. Durante o seguimento não foram diagnosticadas alterações hepáticas ou musculares graves.

\section{Discussão}

Neste estudo, conforme critérios de inclusão adotados, a população estudada apresentou altas taxas de obesidade, tabagismo, HAS e sedentarismo, evidenciando tratar-se de uma população de alto risco para doença cardiovascular. Do ponto de vista de dislipidemias, a população apresentou, além de níveis elevados de colesterol total, LDL-C, índices de Castelli e escores de Framingham altos. Como visto, as mulheres tinham idade mais elevada do que os homens, sugerindo pela média de idade, que uma grande parte dessas mulheres estivesse no período após a menopausa. A predominância de idade maior entre mulheres, em comparação com os homens, é justificada pelos critérios de inclusão (pequeno potencial de engravidar) e pelo fato de fatores de risco, como HAS e hipercolesterolemia manifestarem-se mais tardiamente nas mulheres do que nos homens ${ }^{7,8}$. Ao predomínio da HAS associou-se, como conseqüência, a maior prevalência de hipertrofia ventricular esquerda e maiores índices de Framingham nas mulheres. A maior prevalência de HAS e hipertrofia ventricular esquerda são compatíveis com as altas taxas de AVC descritas para a nossa população ${ }^{2,3}$. Sabidamente, a HAS é um fator de risco mais importante para o AVC do que para a DAC ${ }^{9}$. A hipertrofia ventricular esquerda é também considerada marcador de risco elevado para $\mathrm{AVC}^{10}$. Embora o número de obesos não tenha sido diferente nos grupos, o sedentarismo também prevaleceu nas mulheres. Seguramente, a obesidade associada à ausência de terapia de reposição hormonal para a menopausa nessa população (critério de inclusão neste estudo), influenciaram negativamente o perfil lipídico ${ }^{11,12}$. Na população estudada, foi encontrada tendência a um número maior de manifestações prévias da DAC no sexo masculino. Este resultado é compatível com a história natural desta doença, que mostra incidência mais precoce no sexo masculino ${ }^{8}$. Níveis mais baixos de HDL-C, também, predominaram nos homens. Estudos epidemiológicos mostram que, mesmo após a redução que ocorre nas mulheres, após a menopausa, o HDL-C ainda é mais baixo no sexo masculino ${ }^{13,14}$. A maior prevalência de tabagismo no sexo masculino, assim como níveis mais elevados dos triglicérides, certamente influenciaram estes achados. Níveis menores de HDL-C resultaram em maiores índices de Castelli no sexo masculino. Estes índices são preditores importantes do risco de DAC ${ }^{15}$, sugerindo que, apesar de se tratar de uma população com hipercolesterolemia, com provável predominância de mulheres na pós menopausa, o risco de coronariopatia ainda seja maior no sexo masculino. Estudos epidemiológicos mostram que, apesar do aumento da incidência de DAC no período da pós menopausa, a prevalência desta doença ainda é maior no sexo masculino ${ }^{8}$.

A instituição da terapia dietética e medicamentosa levou à diminuição do peso e melhora da PA e do perfil lipídico. A administração de $10 \mathrm{mg} /$ dia de pravastatina associada à dieta não se acompanhou de efeitos colaterais significativos, sendo bem tolerada em ambos os sexos. À luz dos conhecimentos atuais, o uso das estatinas tornou-se tratamento obrigatório para pacientes de alto risco para a doença coronária ${ }^{16,17}$. O estudo Woscops (prevenção primária da aterosclerose com pravastatina) demonstrou que numa população de homens semelhante à avaliada neste estudo, $\mathrm{o}$ uso de pravastatina reduziu em cerca de $31 \%$ o risco combinado de IM não fatal e de mortalidade por DAC. Além disso, a necessidade de realização de angioplastia coronária ou de cirurgia de revascularização do miocárdio foi diminuído em $27 \%$ com o tratamento, quando comparada ao grupo placebo. Em portadores de manifestação prévia de DAC, os resultados são mais expressivos ainda. Estudos como o $4 \mathrm{~S}^{18}$, o CARE ${ }^{19}$ e, mais recentemente, o LIPID ${ }^{20}$ mostraram redução não só de eventos cardiovasculares e da mortalidade coronária mas, principalmente, redução da mortalidade por todas as causas. Nos dois últimos estudos mostrou-se que o benefício da pravastatina estendeu-se inclusive para pacientes com níveis de colesterol dentro da média da população. Quanto ao custo do tratamento, quando aplicado a populações de alto risco, também se justifica do ponto de vista econômico ${ }^{21,22}$.

Em relação à prevenção da DAC no sexo feminino, existe forte evidência, baseada em estudos populacionais, de que a terapia de reposição hormonal com estrogênios e progestágenos previna esta moléstia após a menopausa ${ }^{23}$. Os achados são mais evidentes nas portadoras da doença já manifesta. Quanto à terapia com estatinas, não existe dúvida de que essas drogas tenham seu papel na prevenção secundária ${ }^{18-20}$. Muito provavelmente o terão também na prevenção primária, principalmente nas mulheres de alto risco. Aguardamos o resultado de estudos controlados que estão em andamento.

Em nosso estudo, os homens tiveram maiores reduções dos índices de Castelli do que as mulheres. Entretanto, não é possível dizer, devido às características deste estudo, se a maior redução dos índices de Castelli refletirá em maior benefício para o sexo masculino. Pelo contrário, existe evidência de estudos como o CARE ${ }^{19}$ de que talvez as mulheres se beneficiem mais na prevenção de eventos cardiovasculares, com tratamento com estatinas, do que os homens. Porém, estes dados ainda necessitam confirmação.

Embora a hipercolesterolemia não seja considerada um fator de risco tão importante para os $\mathrm{AVCs}^{23}$ como a HAS ${ }^{9,23}$, certamente ela exerce seu papel na patogênese desta doença ${ }^{3}$. Um achado importante dos estudos, tanto de prevenção primária como da secundária, é que, além da prevenção 
de DAC, a redução do colesterol reduz, em ambos os sexos, a incidência de AVC isquêmico. Uma recente metanálise ${ }^{25}$ dos estudos disponíveis mostrou redução de $29 \%$ no risco de AVC, $22 \%$ na mortalidade total e $28 \%$ na mortalidade cardiovascular. Não houve aumento na incidência de hemorragia cerebral. Em nossa opinião, este fato justificaria o uso das estatinas associado ao controle da HAS, para a prevenção do AVC em uma população como a avaliada neste estudo.
Mesmo apresentando critérios de seleção bem estabelecidos, este foi um estudo aberto não randomizado. Devemos levar em consideração as suas limitações. Entre elas, variações no perfil lipídico decorrentes da falta de um laboratório central para as determinações dos lípides. Porém, os resultados são compatíveis com levantamentos epidemiológicos em populações semelhantes de nosso meio ${ }^{1} \mathrm{e}$ de outros países ${ }^{26}$.

\section{Referências}

1. Lotufo PA - A mortalidade precoce por doenças crônicas nas capitais de regiões metropolitanas brasileiras.[Tese de Doutorado] São Paulo, Faculdade de Saúde Pública da Universidade de São Paulo, 1996.

2. Lotufo PA - Doenças cardiovasculares no Brasil: por que altas taxas de mortalidade entre mulheres ? Rev Soc Cardiol Estado de São Paulo 1996; 6: 667-71.

3. Lessa I, Bastos CA - Epidemiology of cerebrovascular accidents in the city of Salvador, Bahia, Brazil. PAHO Bulletin 1986; 17: 292-303.

4. Anderson KM, Wilson PWF, Odell PM, Kannel WB - An updated coronary risk profile. Circulation 1991; 83: 356-62.

5. Castelli WP, Garrison RJ, Wilson PWF, Abbot RD, Kalousdian S, Kannel WN Incidence of coronary heart disease and lipoprotein cholesterol levels: the Framingham Heart Study. JAMA 1986; 256: 2835-8.

6. Friedwald WT, Levy RI, Fredrickison DS - Estimation of the concentration of low density lipoprotein cholesterol in plasma, without the use of preparative ultracentrifuge. Clin Chem 1972; 18: 499-552.

7. Manson JE, Colditz GA, Stampfer MJ et al - A prospective study of maturity-onset diabetes mellitus and risk of coronbary heart disease and stroke in women. Arch Intern Med 1991; 265: 627-31.

8. Wenger NK - Epidemiology of coronary heart disease in women. Rev Soc Cardiol Estado de São Paulo 1996; 6: 672-80.

9. Welin L, Svardsudd K, Wilhelmsem L, Larson B, Tibblin G-Analysis of risk factors for stroke in a cohort of men born in 1913. N Eng J Med 1987; 317: 521-6.

10. Koren MJ, Devereux RB, Casale PN, Savage DD, Laragh JH-Relation of left ventricular mass and geometry to morbidity and mortality in uncomplicated essential hypertension. Ann Intern Med 1991; 114: 345-52.

11. Manson JE, Colditz GA, Stampfer MJ et al - A prospective study of obesity and risk of coronary heart disease in women. N Eng J Med 1990; 322: 882-9.

12. Lobo RA - Effects of hormone replacement on lipids and lipoproteins in menopausal women. J Clin Endocrinol Metab 1991; 73: 373-9.

13. Wilson PWF, Christiansen JC, Anderson KM et al - Impact of national guidelines for cholesterol risk factor screening. The Framingham offspring study. JAMA 1989; 262: 41-4.
14. Kannel WB - Range of serum cholesterol values in the population developing coronary artery disease. Am J Cardiol 1995; 76: 69C-77C.

15. Prihoda JS, Illingworth DR - Drug therapy of hyperlipidemia. Curr Prob Cardiol 1992; 17: 549-605.

16. $2^{0}$ Consenso Brasileiro de Dislipidemias. Arq Bras Cardiol 1996; 67: 109-28.

17. Shepherd J, Cobbe S M, Ford I et al - Prevention of coronary heart disease with pravastatin in men with hypercholesterolemia. N Eng J Med 1995; 333 1301-7.

18. Scandinavian Simvastatin Survival Study Group. Randomised trial of cholesterol lowering 4,444 patients with coronary artery disease: Scandinavian Simvastatin Survival Study. Lancet 1994; 344: 1383-9.

19. Sacks FM, Pfeffer MA, Moye LA et al - The effect of pravastatin on coronary events after myocardial infarction in patients with average cholesterol levels. $\mathrm{N}$ Eng J Med 1996; 335: 1001-9.

20. Tonkin A - Long Term Intervention With Pravastatin in Ischemic DiseaseLIPID Study. Apresentado no Congresso da American Heart Association, 1997.

21. Shepherd J, for the West of Scotland Prevention Study Economic Analysis Group. The Cost effectiveness of preventing initial coronary events with pravastatin. Results of the West of Scotland Coronary Prevention Study economic analysis. J Amer Coll Cardiol 1997; 29 suppl A: 168A.

22. Ashraf T, Hay JW, Pitt B et al - Cost effectiveness of pravastatin in secondary prevention of coronary artery disease. Am J Cardiol 1996; 78: 409-14.

23. Gray J-Clinical evidence for postmenopausal hormone replacement therapy. Rev Soc Cardiol Estado de São Paulo 1996; 6: 718-23.

24. Feinberg WM, Albers GW, Barnett JM et al - Guidelines for the management of transient ischemic attacks. Stroke 1994; 25: 1320-35.

25. Herbert PR, Graziano JM, Chan KS, Hennekens CH-Cholesterol lowering with statin drugs, risk of stroke, and total mortality: an overview of randomized trials. JAMA 1997; 278: 313-21.

26. Miettinen TA, Pyorala K, Olsson AG et al - Cholesterol-lowering therapy in women and elderly patients with myocardial infarction or angina pectoris. Findings from the Scandinavian Simvastatin Survival Study. Circulation 1997; 96 : 4211-8. 